\title{
Chronic rhinosinusitis is associated with higher prevalence and severity of bronchiectasis in patients with COPD
}

This article was published in the following Dove Press journal:

International Journal of COPD

20 February 2017

Number of times this article has been viewed

\author{
Xia Yang' \\ Yali Xu' \\ Jianmin Jin' \\ Ruimin $\mathrm{Li}^{\prime}$ \\ Xiaofang Liu' \\ Yongchang Sun ${ }^{1,2}$ \\ 'Department of Respiratory Medicine, \\ Beijing Tongren Hospital, Capital \\ Medical University, ${ }^{2}$ Department \\ of Respiratory and Critical Care \\ Medicine, Peking University Third \\ Hospital, Beijing, People's Republic \\ of China
}

Correspondence: Yongchang Sun Department of Respiratory Medicine, Beijing Tongren Hospital, Capital Medical University, I Dongjiaominxiang, Dongcheng, Beijing 100730, People's Republic of China

Tel +86I56 II 963697

Email suny@bjmu.edu.cn
Background and purpose: Bronchiectasis revealed by high-resolution computed tomography (HRCT) is common in chronic obstructive pulmonary disease (COPD), but the causes and risk factors remain to be determined. Chronic rhinosinusitis (CRS) is closely associated with bronchiectasis or COPD, but whether it is associated with comorbid bronchiectasis in COPD (COPD-Bx) is unknown.

Patients and methods: Patients with stable COPD were enrolled consecutively and evaluated for the presence of CRS by questionnaire and paranasal sinus computed tomography. The presence and severity of bronchiectasis on lung HRCT were evaluated by the Smith and severity scores. COPD symptoms were evaluated by COPD Assessment Test (CAT) and Modified British Medical Research Council Questionnaire. The sputum cell differentials and concentrations of interleukin (IL)-6, IL-8, IL-5, matrix metalloproteinases-9 (MMP-9), and tissue inhibitor of matrix metalloproteinases-1 were measured.

Results: We enrolled 136 patients with stable COPD, of which 66 (48.5\%) were diagnosed with CRS according to the European Position Paper on Rhinosinusitis and Nasal Polyps (EP ${ }^{3} \mathrm{OS}$ ) criteria. The prevalence of bronchiectasis was $57.6 \%$ in patients with CRS, but $37.1 \%$ in those without CRS $(P=0.017)$. COPD-Bx patients with CRS showed a significantly higher severity score of bronchiectasis than those without CRS $(P=0.034)$. COPD patients with CRS had a higher percentage of eosinophils, higher levels of IL-8, IL-6, and MMP-9 in sputum as compared to those without CRS. In COPD-Bx patients with CRS, the percentage of eosinophils and the levels of IL-6 and MMP-9 in sputum were increased as compared to those without CRS. In all the subjects, Sino-Nasal Outcome Test-20 correlated with CAT score $(r=0.315, P<0.01)$ and in COPD patients with CRS, LundMacKay scores correlated with forced expiratory volume in $1 \mathrm{~s}$ ( $\%$ pred $)(r=-0.251, P<0.05)$.

Conclusions: CRS was associated with COPD-Bx and this was probably due to increased airway inflammation.

Keywords: bronchiectasis, COPD, chronic rhinosinusitis

\section{Introduction}

With the increasing use of chest high-resolution computed tomography (HRCT) in the assessment of patients with chronic obstructive pulmonary disease (COPD), previously unrecognized radiographic bronchiectasis has been identified. ${ }^{1}$ Studies showed that 15\%-30\% of patients diagnosed with COPD had bronchiectasis by CT scanning, and the prevalence could even reach $50 \%$ in patients with severe COPD. ${ }^{2-4}$ The presence of bronchiectasis in patients with COPD predicted worse health status and increased mortality, ${ }^{5}$ and thus is regarded as a comorbidity of COPD, although the underlying mechanism is currently unknown. 
Chronic rhinosinusitis (CRS) is a common upper airway disease, which is characterized by chronic inflammation of nasal mucosa and paranasal sinuses. The estimated prevalence of CRS is $10.9 \%$ in Europe and $16 \%$ in the USA. ${ }^{6,7}$ In China, a cross-sectional survey showed that the overall prevalence of self-reported CRS was $8.2 \%$ among adults aged $15-75$ years. ${ }^{8}$

Epidemiological and physiological studies have demonstrated that lower and upper airway diseases often coexist, which has led to the concept of the "united airways". ${ }^{9}$ Both allergic and nonallergic lower airway diseases were found to be associated with significant sinonasal inflammation and symptoms. ${ }^{10}$ In patients with a primary diagnosis of bronchiectasis, the prevalence of CRS was high, and CRS had a considerable impact on the quality of life (QoL) of the patients and was correlated with the severity of bronchiectasis by CT scoring. ${ }^{11-13}$ All these findings suggest that CRS may be involved in or share the pathogenesis of bronchiectasis, although the exact links between them remain speculative.

Recent studies also reported high prevalence of sinonasal symptoms in COPD, ranging from $33 \%$ to $88 \%$, and patients with severe to very severe COPD had significantly worse impairment of health status because of upper airway symptoms than those with mild-to-moderate disease. ${ }^{14-17}$ However, whether CRS is associated with comorbid bronchiectasis in COPD (COPD-Bx) remains unanswered. The aim of this study was to explore the association between CRS and bronchiectasis in COPD patients. We hypothesized that the concept of "united airways" also applied to bronchiectasis associated with COPD.

\section{Materials and methods Study subjects}

Adult patients with stable COPD visiting Beijing Tongren Hospital, Capital Medical University, were consecutively enrolled between March 2015 and February 2016. COPD was diagnosed according to Global Initiative for Chronic Obstructive Lung Disease (GOLD) criteria. ${ }^{1}$ Pulmonary function tests were performed according to international standards by two trained and experienced technicians. Severity of airflow obstruction was evaluated according to the 2014 GOLD classification. All subjects were required to either be a current smoker or a past smoker. Subjects with a primary diagnosis of bronchiectasis, asthma or any other significant respiratory diseases were excluded. The study was approved by the Ethics Committee of Beijing Tongren Hospital, Capital Medical University (TRECKT2008-14), and all subjects provided informed written consent.

\section{Clinical data and COPD assessment}

Clinical data were collected, including demographic characteristics, smoking history, duration of COPD symptoms, nasal symptoms, and previous diagnosis and treatment of sinusitis. Dyspnea was evaluated using the Modified British Medical Research Council (mMRC) Questionnaire, and overall symptoms were evaluated by the COPD Assessment Test (CAT).

\section{Chest HRCT scanning}

Chest HRCT was performed using a 64-row, multipledetector CT scanner (Philips Company, Amsterdam, the Netherlands). The diagnosis of bronchiectasis was based on criteria reported by Naidich et $\mathrm{al}^{18}: 1$ ) lack of tapering of bronchi, 2) dilation of bronchi when the luminal diameter was larger than that of the adjacent pulmonary artery, or 3) visualization of the peripheral bronchi within $1 \mathrm{~cm}$ of the costal pleural surface or adjacent mediastinal pleural surface. The radiographic extent of bronchiectasis was assessed using the Smith method. ${ }^{5,19}$ The extent of bronchiectasis in each lobe was graded as follows: 0 , if there was no evidence of bronchiectasis; $1,<25 \%$ of the bronchi were abnormal; 2 , $25 \%-49 \%$ of the bronchi were abnormal; $3,50 \%-74 \%$ of the bronchi were abnormal; and $4,75 \%$ or more of the bronchi were abnormal. The lingula was scored as a separate lobe. The total Smith score ranged from 0 to 24 . The severity of bronchiectasis in each lobe was graded by the Bhalla scoring system, ${ }^{20}$ using a scale from 0 to $3: 0$, no involvement; 1 , mild, luminal diameter slightly greater than diameter of adjacent blood vessel; 2, moderate, lumen 2-3 times the diameter of adjacent vessel; and 3, severe, lumen $>3$ times the diameter of adjacent vessel. Small cylindrical bronchiectasis visible in only a single pulmonary segment was not considered, because this can appear in a significant percentage of the healthy population, as previously reported. ${ }^{21}$ The images were assessed by two professional radiologists blindly. When the two independent radiologists disagreed in their evaluation, the scores were decided together after discussion.

\section{Evaluation of CRS}

CRS was diagnosed based on the European Position Paper on Rhinosinusitis and Nasal Polyps (EP $\left.{ }^{3} \mathrm{OS}\right)$ criteria by clinical symptoms and sinus CT features ${ }^{22}: 1$ ) For the clinical diagnosis of sinusitis, major and minor criteria were applied. Major symptoms were nasal obstruction/blockage and nasal or post-nasal discharge/purulence. Minor symptoms included headache, facial pain/pressure/fullness, and hyposmia/anosmia. Patients were classified as having CRS if they reported usual presence of at least two of these 
symptoms for $>12$ consecutive weeks, and at least one of the two major symptoms was present. 2) CT features included mucosal changes of ostiomeatal complex and/or sinuses. The presence/absence of nasal polyps could not be ascertained because nasal endoscopy or tissue biopsy was not performed. The paranasal sinus computed tomography (PNS-CT) scans of the subjects were obtained using a Philips Brilliance CT scanner. The radiographic severity of CRS was assessed using the Lund-MacKay CT staging system (0-24) by two professional radiologists blindly. ${ }^{23}$ The minimal possible score " 0 " was considered as normal or negative; any score $>0$ was considered as abnormal or positive. Patients also filled in the Sino-Nasal Outcome Test-20 (SNOT-20) questionnaire, which is a validated disease-specific QoL questionnaire for rhinosinusitis. ${ }^{24}$ The SNOT-20 comprises 20 items and each item is graded from 0 to 3 , with total scores ranging from 0 to 60 . Higher scores represent a worse impairment of health status because of upper airway symptoms.

\section{Sputum sample processing}

Sputum induction was performed in all subjects according to the method described by Paggiaro et al. ${ }^{25}$ The sputum samples were kept on ice until processed, usually within $1 \mathrm{~h}$. Sputum was weighed and mixed with $0.1 \%$ dithiothreitol in a ratio of $4: 1$. The sample was filtered to increase homogenization and centrifuged at $2,000 \times g$ at $4^{\circ} \mathrm{C}$ for $10 \mathrm{~min}$. The supernatants were harvested and stored at $-80^{\circ} \mathrm{C}$ until analysis. Cytospin slides were prepared and stained with Wright Giemsa staining for the differential cell counts.

\section{Measurement of inflammatory mediators}

The levels of interleukin (IL)-6, IL-8, and IL-5 in the supernatants of sputum were determined by means of Cytometric Bead Array Flex System (Becton Dickinson [BD] Pharmingen, San Jose, CA, USA) according to the manufacturer's instructions. The detection limits of IL-6, IL-8, and IL-5 were $10 \mathrm{pg} / \mathrm{mL}$. The concentrations of matrix metalloproteinases-9 (MMP-9) and tissue inhibitors of matrix metalloproteinases-1 (TIMP-1) in supernatants of sputum were measured with commercial ELISA kits (R\&D Systems, Inc., Minneapolis, MN, USA). The detection limits of MMP-9 and TIMP-1 were $31.3 \mathrm{pg} / \mathrm{mL}$. A zero value was assigned when the concentration of inflammatory mediators was below the detection limit.

\section{Statistical analysis}

All statistical analyses were conducted using a statistical software package (version 21.0; Statistics Package for the
Social Sciences [SPSS], Inc., Chicago, IL, USA). Data were expressed as the mean \pm standard deviation (for normal distribution parameters) or as median and 25 th-75th percentiles (for abnormal distribution parameters). Comparisons of continuous data between two groups were performed by $t$-test (for normal distribution parameters) and Mann-Whitney $U$-test (for abnormal distribution parameters). Categorical variables between different groups were analyzed by $\chi^{2}$ test. Spearman's correlations were used for correlation analysis. $P$-values $<0.05$ were considered statistically significant.

\section{Results \\ Demographic characteristics}

One hundred and thirty-six patients with COPD were enrolled for this study, among whom 17 (12.5\%), 68 (50.0\%), 43 $(31.6 \%)$, and $8(5.9 \%)$ were in GOLD stages I, II, III, and IV, respectively. The mean age was $71.3 \pm 11.1$ years, and $83.8 \%$ were male. A total of $66.2 \%(90 / 136)$ of the subjects were current smokers (Table 1).

\section{Prevalence of bronchiectasis and CRS in patients with COPD}

Chest HRCT revealed that $47.1 \%(64 / 136)$ of the COPD patients had signs of bronchiectasis which was commonly seen in the right lower lobe $(59.4 \%)$, followed by right middle lobe $(57.8 \%)$, left lower lobe (48.4\%), right upper lobe (40.6\%), left upper lobe (37.5\%), and lingula (29.7\%), and bilateral lesions were common. Most patients showed cylindrical bronchiectasis $(87.5 \%)$, while a minority of patients had mixed bronchiectasis $(12.5 \%)$.

According to the $\mathrm{EP}^{3} \mathrm{OS}$ criteria, 48.5\% (66/136) of the COPD patients were diagnosed to have CRS. Nasal obstruction, nasal or post-nasal discharge, headache/facial pain, and anosmia were present in $79.5 \%, 94.9 \%, 23.1 \%$, and $48.7 \%$ of the patients, respectively. By PNS-CT scanning, the predilection sites of CRS were maxillary (68.2\%), anterior ethmoid (53.0\%), posterior ethmoid (33.3\%), sphenoid (24.2\%), frontal $(22.7 \%)$, and ostiomeatal complex (7.6\%), and bilateral lesions were common.

\section{Characteristics of COPD patients with and without CRS}

Clinical characteristics of COPD patients with and without CRS are shown in Table 1. Subjects with CRS had significantly lower forced expiratory volume in $1 \mathrm{~s}$ (\% pred), longer duration of symptoms, and higher scores of CAT and mMRC. There were more females in COPD patients with CRS as compared to those without CRS. In COPD patients 
Table I Characteristics of COPD patients with and without CRS

\begin{tabular}{|c|c|c|c|c|}
\hline Item & All COPD patients & COPD patients with CRS & COPD patients without CRS & $P$-value \\
\hline Subjects (n) & 136 & 66 & 70 & \\
\hline Age (years) & $71.3 \pm 11.1$ & $72.0 \pm 11.0$ & $70.7 \pm 11.2$ & 0.501 \\
\hline Male/female (n) & $5.2(114 / 22)$ & $3.4(5|/| 5)$ & $9(63 / 7)$ & $0.025^{*}$ \\
\hline Smoking, pack-years & $48.8(30.0,60.0)$ & $49.8(30.0,60.0)$ & $45.8(30.0,60.5)$ & 0.648 \\
\hline Symptom duration (years) & $8.5(4.0,20.0)$ & $10.0(5.0,20.0)$ & $5.0(3.0,12.8)$ & $0.011 *$ \\
\hline $\mathrm{FEV}_{\text {, }}(\%$ predicted $)$ & $57.0 \pm 18.1$ & $53.4 \pm 18.1$ & $60.4 \pm 17.6$ & $0.025^{*}$ \\
\hline $\mathrm{FEV}_{\mathrm{I}} / \mathrm{FVC}(\%)$ & $59.3(48.7,64.8)$ & $57.1(44.7,63.3)$ & $61.2(53.5,65.8)$ & 0.085 \\
\hline BMI $\left(\mathrm{kg} / \mathrm{m}^{2}\right)$ & $24.5 \pm 4.0$ & $24.5 \pm 4.3$ & $24.5 \pm 3.7$ & 0.915 \\
\hline \multicolumn{5}{|l|}{ GOLD stage } \\
\hline l & $17(12.5 \%)$ & $7(10.6 \%)$ & $10(14.3 \%)$ & \\
\hline II & $68(50.0 \%)$ & $28(42.4 \%)$ & $40(57.1 \%)$ & \\
\hline III & $43(31.6 \%)$ & 26 (39.4\%) & $17(24.3 \%)$ & \\
\hline IV & $8(5.9 \%)$ & $5(7.6 \%)$ & $3(4.3 \%)$ & \\
\hline CAT & $16.0(13.0,21.0)$ & $19.0(14.0,23.3)$ & $14.5(13.0,19.0)$ & $0.00 I^{* *}$ \\
\hline $\mathrm{mMRC}$ & $1.0(1.0,2.0)$ & $2.0(1.0,3.0)$ & $1.0(0.0,2.0)$ & $0.010^{*}$ \\
\hline SNOT-20 & $3.0(1.0,4.0)$ & $4.0(4.0,5.3)$ & I.0 $(1.0,2.0)$ & $0.000 * * *$ \\
\hline
\end{tabular}

Notes: Data presented as mean $\pm S D$, median with $25-75$ th percentiles, or $n(\%)$. $* P<0.05 ; * * P<0.01 ; * * * P<0.001$.

Abbreviations: BMI, body mass index; CAT, COPD assessment test; COPD, chronic obstructive pulmonary disease; CRS, chronic rhinosinusitis; FEV , forced expiratory volume in I s; FVC, forced vital capacity; GOLD, Global Initiative for Chronic Obstructive Lung Disease; mMRC, modified Medical Research Council; SNOT-20, Sino-Nasal Outcome Test-20; SD, standard deviation.

with CRS, the Lund-MacKay scores correlated with FEV (\% pred) $(r=-0.251, P<0.05)$. In all the COPD patients, the SNOT-20 correlated with the CAT scores $(r=0.315, P<0.01$; Figure 1).

\section{Prevalence and severity of bronchiectasis} in COPD patients with and without CRS In COPD patients with CRS $(n=66), 57.6 \%(38 / 66)$ showed comorbid bronchiectasis, while in those without CRS $(\mathrm{n}=70)$, $37.1 \%(26 / 70)$ did; the difference being significant $\left(\chi^{2}=5.693\right.$, $P=0.017)$. The CT score was used to evaluate the extent and severity of bronchiectasis in COPD patients. The scores of bronchiectasis between COPD-Bx patients with and without
CRS are shown in Table 2. The severity score of bronchiectasis in COPD-Bx patients with CRS was significantly higher than those without CRS, while the Smith score, that is, the radiographic extent of bronchiectasis, was comparable between COPD-Bx patients with and without CRS.

\section{Airway inflammatory cells and mediators in COPD patients with and without CRS}

To investigate the underlying mechanisms of the association between CRS and bronchiectasis in COPD, we examined the numbers of inflammatory cells and measured the concentrations of cytokines or mediators related to airway neutrophilic or eosinophilic inflammation in sputum. We also
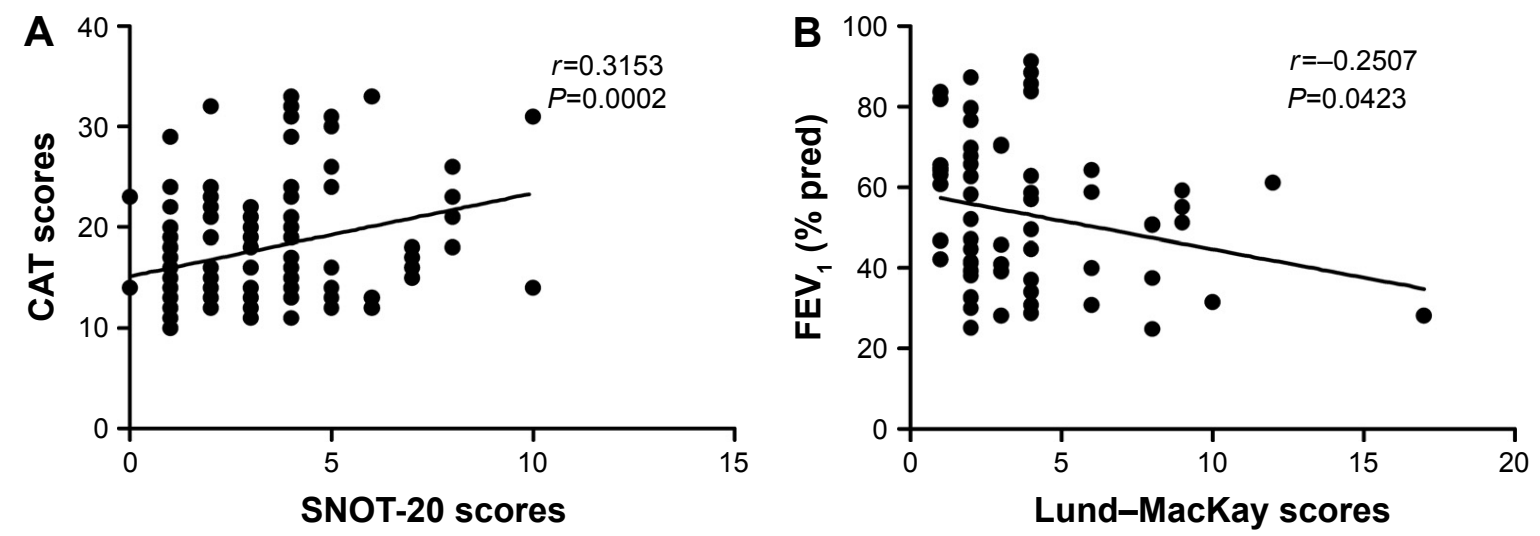

Figure I The results of correlation analysis.

Notes: (A) The correlation between SNOT-20 and CAT scores in all subjects. (B) The correlation between Lund-MacKay scores and FEV (\% pred) in COPD patients with CRS.

Abbreviations: CAT, COPD assessment test; COPD, chronic obstructive pulmonary disease; CRS, chronic rhinosinusitis; FEV , forced expiratory volume in I s; SNOT-20, Sino-Nasal Outcome Test-20. 
Table 2 The prevalence and the severity of bronchiectasis in COPD patients with and without CRS

\begin{tabular}{llll}
\hline Item & $\begin{array}{l}\text { COPD } \\
\text { patients with } \\
\text { CRS (n=66) }\end{array}$ & $\begin{array}{l}\text { COPD patients } \\
\text { without CRS }\end{array}$ & $\begin{array}{l}\text { P-value } \\
(\mathbf{n}=70)\end{array}$ \\
\hline COPD-Bx subjects (\%) & $38(57.6 \%)$ & $26(37.1 \%)$ & $0.017^{*}$ \\
The severity score & $3.0(2.0,5.0)$ & $2.0(2.0,3.0)$ & $0.034^{*}$ \\
Smith score & $6.0(4.0,10.0)$ & $4.9(3.0,8.0)$ & 0.179 \\
\hline
\end{tabular}

Notes: Data are presented as median with 25th-75th percentiles or $n$ (\%). The severity score indicates the radiological score of bronchiectasis severity; the Smith score indicates the radiological score of bronchiectasis extent. ${ }^{*}<0.05$.

Abbreviations: COPD, chronic obstructive pulmonary disease; COPD-Bx, comorbid bronchiectasis in COPD; CRS, chronic rhinosinusitis.

measured the sputum level of MMP-9 and TIMP-1, which are believed to be involved in airway damage and remodeling, a feature of bronchiectasis. The level of IL-5 was below the detection limit in nearly all sputum samples and, therefore, was not included in this analysis. The percentage of sputum eosinophils, and the sputum levels of IL-6, IL-8, and MMP-9 were increased in COPD patients with CRS as compared with those without CRS. The results are shown in Table 3.

\section{Airway inflammatory cells and mediators in COPD-Bx patients with and without CRS}

Because the severity score of bronchiectasis was higher in COPD-Bx patients with CRS as compared to those without CRS, we asked whether this was associated with enhanced features of airway inflammation. Our results showed that the percentage of eosinophils and the levels of IL-6 and MMP-9 in sputum were increased in COPD-Bx patients with CRS as compared to COPD-Bx patients without CRS. The percentage of sputum neutrophils, macrophages, and the levels of sputum IL-8 did not differ between COPD-Bx patients with and without CRS. The results are shown in Table 4.

\section{Discussion}

We have shown for the first time to our knowledge that in a well-defined cohort of smokers with COPD, CT bronchiectasis was more prevalent and severe in patients with CRS, which was associated with increased airway eosinophils, IL-6, IL-8, and MMP-9. COPD patients with CRS had more severe impairment of pulmonary function, and higher scores of CAT and mMRC. We also found that Lund-MacKay scores correlated with $\mathrm{FEV}_{1}$ (\% pred), implying that there may be a link between the severity of CRS and lung function decline in COPD.

In our study, the prevalence of CRS defined by the $\mathrm{EP}^{3} \mathrm{OS}$ criteria in COPD patients, mostly with moderate-to-severe disease, was $48.5 \%$, which was consistent with the results of most studies in patients with COPD. ${ }^{14,15}$ The prevalence of CT-defined bronchiectasis in our COPD patients was $47.1 \%$, also similar to previous findings. ${ }^{2-4}$ A novel finding of our study was that the prevalence of bronchiectasis in COPD patients with CRS was significantly higher than that in those without CRS, and interestingly, COPD-Bx subjects with CRS showed a significantly higher severity score of bronchiectasis than those without CRS. Our results suggest that CRS may influence the pathogenesis or progression of bronchiectasis in COPD. While "primary" bronchiectasis is mostly due to infectious insults of the lower airways (postinfectious bronchiectasis) ${ }^{26}$ the pathogenesis of bronchiectasis in COPD is probably more complicated. It is suggested that persistent airway inflammation in COPD with ensuing tissue injury and remodeling may induce the occurrence of bronchiectasis., ${ }^{2,4}$ Our study lends support to that hypothesis.

We found that airway inflammatory profiles between COPD patients with and without CRS differed. The percentage of sputum eosinophils and the sputum levels of IL-6, IL-8, and MMP-9 were increased in COPD patients with CRS as compared to those without CRS. The data also suggest that

Table 3 Sputum inflammatory cells and mediators in COPD patients with and without CRS

\begin{tabular}{|c|c|c|c|}
\hline Item & COPD subjects with CRS $(n=66)$ & COPD subjects without CRS $(n=70)$ & $P$-value \\
\hline Neutrophils (\%) & $26.88(8.44,54.69)$ & $20.58(5.53,51.34)$ & 0.118 \\
\hline Lymphocytes (\%) & $3.56(2.4 I, 7.32)$ & $4.35(2.40,8.99)$ & 0.519 \\
\hline Macrophages (\%) & $58.68(32.17,81.24)$ & $73.73(43.96,81.82)$ & 0.095 \\
\hline Eosinophils (\%) & $0.55(0.00,3.20)$ & $0.00(0.00,1.10)$ & $0.004 * *$ \\
\hline IL-6 (pg/mL) & $492.32(68.96,|46| .46)$ & $282.25(43.65,65 I .29)$ & $0.046 *$ \\
\hline IL-8 (ng/mL) & $33.31(10.30,112.24)$ & $23.65(6.90,40.90)$ & $0.027^{*}$ \\
\hline MMP-9 (ng/mL) & $765.10(340.99,17 \mid 4.14)$ & $631.60(220.89,902.27)$ & $0.042^{*}$ \\
\hline TIMP-I (ng/mL) & $704.16(239.32,1227.25)$ & $562.65(154.28,1253.76)$ & 0.467 \\
\hline MMP-9/TIMP-I & I.5। $(0.86,2.59)$ & $\mathrm{I} .3 \mathrm{I}(0.73,2.5 \mathrm{I})$ & 0.438 \\
\hline
\end{tabular}

Notes: Data are presented as median with 25 th-75th percentiles. $* P<0.05 ; * * P<0.01$.

Abbreviations: COPD, chronic obstructive pulmonary disease; CRS, chronic rhinosinusitis; IL, interleukin; MMP-9, matrix metalloproteinases-9; TIMP-I, tissue inhibitors of matrix metalloproteinases-I. 
Table 4 Sputum inflammatory cells and mediators in COPD-Bx patients with and without CRS

\begin{tabular}{llll}
\hline Item & COPD-Bx subjects with CRS (n=38) & COPD-Bx subjects without CRS (n=26) & P-value \\
\hline Neutrophils (\%) & $43.62(8.86,67.13)$ & $38.13(3.54,57.63)$ & 0.363 \\
Lymphocytes (\%) & $3.37(2.41,6.47)$ & $3.36(2.39,7.64)$ & 0.875 \\
Macrophages (\%) & $45.8 I(23.44,79.45)$ & $58.13(38.66,84.02)$ & 0.229 \\
Eosinophils (\%) & $2.10(0.00,3.20)$ & $0.00(0.00,2.08)$ & $0.036^{*}$ \\
IL-6 $(\mathrm{pg} / \mathrm{mL})$ & $492.32(96.68,1592.89)$ & $130.70(41.93,645.44)$ & $0.037^{*}$ \\
IL-8 $(\mathrm{ng} / \mathrm{mL})$ & $30.82(12.07,73.4 I)$ & $23.71(6.37,42.47)$ & 0.097 \\
MMP-9 $(\mathrm{ng} / \mathrm{mL})$ & $851.70(544.04,1837.04)$ & $687.14(221.95,870.33)$ & $0.025^{*}$ \\
TIMP-I $(\mathrm{ng} / \mathrm{mL})$ & $822.10(446.99,1227.25)$ & $655.57(136.45,1667.93)$ & 0.575 \\
MMP-9/TIMP-I & $1.36(0.83,2.27)$ & $0.94(0.45,2.82)$ & 0.353 \\
\hline
\end{tabular}

Notes: Data are presented as median with 25 th-75th percentiles. $* P<0.05$.

Abbreviations: COPD, chronic obstructive pulmonary disease; COPD-Bx, comorbid bronchiectasis in COPD; CRS, chronic rhinosinusitis; IL, interleukin; MMP-9, matrix metalloproteinases-9; TIMP-I, tissue inhibitors of matrix metalloproteinases-I.

CRS may play a role in aggravating lower airway inflammation in COPD. Sputum eosinophils and the levels of IL-6 and MMP-9 were increased in COPD-Bx patients with CRS, who had more severe clinical disease as compared to those without CRS.

Increased levels of neutrophil chemoattractant protein IL-8 and the proinflammatory cytokine IL- 6 in the airways of COPD patients have been correlated with the decline of lung function and severity of disease. ${ }^{27-29}$ These cytokines were also increased in nasal secretions and peripheral blood of patients with CRS. ${ }^{30}$ Hurst et al found that IL-8 in nasal secretion was increased in stable COPD and related to that in the sputum, ${ }^{31}$ suggesting an inflammatory link between upper and lower airway diseases. Elevated levels of IL-6 and IL-8 in bronchoalveolar lavage fluid have also been reported in studies on "primary" bronchiectasis, ${ }^{32,33}$ supporting a role of these cytokines in airway inflammation of bronchiectasis. We did not find any difference in sputum IL-8 and neutrophils in COPD-Bx patients with and without CRS, suggesting that the association of CRS with severity of bronchiectasis in COPD needs to be explained by other mechanisms.

The airway inflammation in COPD is complex. Although it is believed to be predominantly neutrophilic, recent studies have revealed eosinophilic inflammation in the airways of a proportion of patients with COPD both in stable stage and in acute exacerbation. ${ }^{34,35}$ Our recent studies have found a higher prevalence of increased serum total $\mathrm{IgE}$ in patients with $\mathrm{COPD}^{36}$ and an association of serum total $\operatorname{IgE}$ with the extent of COPD-Bx. ${ }^{37}$ Much earlier studies also found evidence of eosinophilic activation in patients with "primary" bronchiectasis or with cystic fibrosis, ${ }^{38,39}$ suggesting that eosinophil-derived granular proteins were involved in bronchiectatic tissue destruction. Here, we found that sputum eosinophils were increased in COPD patients with CRS compared to those without CRS, and more notably, higher in COPD-Bx patients with CRS as compared to COPD-Bx patients without CRS. Considering that both allergy and bacterial infection are involved in the pathogenesis of CRS, ${ }^{40}$ these results suggest a potential role for Th2 inflammatory mechanisms in the pathogenesis of bronchiectasis in COPD. The role of bacterial colonization and repeated infections also needs further investigation.

Airway damage and remodeling are important features of bronchiectasis. Previous studies in bronchiectasis patients had found that sputum MMP-9 and MMP-9/TIMP-1 ratio were increased and correlated with the severity of bronchiectasis. ${ }^{41}$ In our study, sputum MMP-9 was significantly increased in COPD patients with CRS, but the MMP-9/TIMP-1 ratio was not different between groups, suggesting that MMPs, in addition to MMP-9, may also be involved in COPD-Bx.

Our study has several limitations. 1) According to $\mathrm{EP}^{3} \mathrm{OS}, \mathrm{CRS}$ with and without nasal polyps (eg, CRSwNP and CRSsNP) are two important subtypes. Nasal endoscopy was not performed in our study, and hence CRSwNP and CRSsNP could not be differentiated. 2) We did not examine the inflammatory cells and mediators/cytokines in the nasal secretions and the blood of the patients and, therefore, a correlation of the inflammatory profiles of the upper and lower airways could not be established, and we could not conclude whether CRS and comorbid bronchiectasis are associated with enhanced systemic inflammation. 3) Because of the cross-sectional nature of the study, we did not explore the impact of CRS and/or comorbid bronchiectasis on important outcomes of COPD, such as exacerbations.

\section{Conclusion}

In conclusion, we found that COPD patients with CRS had a higher prevalence and severity of comorbid bronchiectasis, 
which was associated with enhanced inflammation of the lower airways. These results support the "united airways" concept in the pathogenesis of comorbid bronchiectasis in patients with COPD.

\section{Acknowledgment}

The authors acknowledge financial support from the National Natural Science Foundation of China (81470239).

\section{Author contributions}

All authors contributed toward data analysis, drafting and critically revising the paper, gave final approval of the version to be published, and agree to be accountable for all aspects of the work.

\section{Disclosure}

The authors report no conflicts of interest in this work.

\section{References}

1. Global Strategy for the Diagnosis, Management, and Prevention of COPD, Global Initiative for Chronic Obstructive Lung Disease (GOLD); 2014. Available from: http://www.goldcopd.org. Accessed November 16, 2014.

2. Patel IS, Vlahos I, Wilkinson TM, et al. Bronchiectasis, exacerbation indices, and inflammation in chronic obstructive pulmonary disease. Am J Respir Crit Care Med. 2004;170(4):400-407.

3. King PT. The pathophysiology of bronchiectasis. Int J Chron Obstruct Pulmon Dis. 2009;4:411-419.

4. Du QX, Jin JM, Liu XF, Sun YC. Bronchiectasis as a comorbidity of chronic obstructive pulmonary disease: a systematic review and metaanalysis. PLoS One. 2016;11(3): 0150532.

5. Martinez-Garcia MA, de la Rosa Carrillo D, Soler-Cataluna JJ, et al. Prognostic value of bronchiectasis in patients with moderate to severe chronic obstructive pulmonary disease. Am J Respir Crit Care Med. 2013;187(8):823-831.

6. Hastan D, Fokkens WJ, Bachert C, et al. Chronic rhinosinusitis in Europe-an underestimated disease. A GA²LEN study. Allergy. 2011;66(9): 1216-1223.

7. Pleis JR, Ward BW, Lucas JW. Summary health statistics for U.S. adults: national health interview survey, 2009. Vital Health Stat 10. 2010;(249):1-207.

8. Shi JB, Fu QL, Zhang H, et al. Epidemiology of chronic rhinosinusitis: results from a cross-sectional survey in seven Chinese cities. Allergy. 2015;70(5):533-539.

9. Passalacqua G, Ciprandi G, Canonica GW. The nose-lung interaction in allergic rhinitis and asthma: united airways disease. Curr Opin Allergy Clin Immunol. 2001;1(1):7-13.

10. Hens G, Vanaudenaerde BM, Bullens DM, et al. Sinonasal pathology in nonallergic asthma and COPD: "united airway disease" beyond the scope of allergy. Allergy. 2008;63(3):261-267.

11. Guilemany JM, Angrill J, Alobid I, et al. United airways again: high prevalence of rhinosinusitis and nasal polyps in bronchiectasis. Allergy. 2009;64(5):790-797.

12. Guan WJ, Gao YH, Li HM, Yuan JJ, Chen RC, Zhong NS. Impacts of co-existing chronic rhinosinusitis on disease severity and risks of exacerbations in Chinese adults with bronchiectasis. PLoS One. 2015; 10(9): $\mathrm{e} 0137348$

13. Guilemany JM, Angrill J, Alobid I, et al. United airways: the impact of chronic rhinosinusitis and nasal polyps in bronchiectasic patient's quality of life. Allergy. 2009;64(10):1524-1529.
14. Montnemery P, Svensson C, Adelroth E, et al. Prevalence of nasal symptoms and their relation to self-reported asthma and chronic bronchitis/ emphysema. Eur Respir J. 2001;17(4):596-603.

15. Kelemence A, Abadoglu O, Gumus C, Berk S, Epozturk K, Akkurt I. The Frequency of chronic rhinosinusitis/nasal polyp in COPD and its effect on the severity of COPD. COPD. 2011;8(1):8-12.

16. Hurst JR, Wilkinson TM, Donaldson GC, Wedzicha JA. Upper airway symptoms and quality of life in chronic obstructive pulmonary disease. Respir Med. 2004;98(8):767-770.

17. Roberts NJ, Lloyd-Owen SJ, Rapado F, et al. Relationship between chronic nasal and respiratory symptoms in patients with COPD. Respir Med. 2003;97(8):909-914.

18. Naidich DP, McCauley DI, Khouri NF, Stitik FP, Siegelman SS. Computed tomography of bronchiectasis. J Comput Assist Tomogr. 1982;6(3):437-444

19. Smith IE, Jurriaans E, Diederich S, Ali N, Shneerson JM, Flower CD. Chronic sputum production: correlation between clinical features and findings on high resolution computed tomographic scanning of the chest. Thorax. 1996;51(9):914-918.

20. Bhalla M, Turcios N, Aponte V, et al. Cystic fibrosis: scoring system with thin-section CT. Radiology. 1991;179(3):783-788.

21. Lynch DA, Newell JD, Tschomper BA, Cink TM, Newman LS, Bethel R. Uncomplicated asthma in adults: comparison of CT appearances of the lungs in asthma and healthy subjects. Radiology. 1993;188(3):829-833.

22. Fokkens WJ, Lund VJ, Mullol J, et al. EPOS 2012: European position paper on rhinosinusitis and nasal polyps 2012. A summary for otorhinolaryngologists. Rhinology. 2012;50(1):1-12.

23. Lund VJ, Mackay IS. Staging in rhinosinusitis. Rhinology. 1993; 31(4):183-184.

24. Piccirillo JF, Merritt MG Jr, Richards ML. Psychometric and clinimetric validity of the 20-item sino-nasal outcome test (SNOT-20). Otolaryngol Head Neck Surg. 2002;126(1):41-47.

25. Paggiaro PL, Chanez P, Holz O, et al. Sputum induction. Eur Respir J. 2002;37:3s-8s.

26. Suarez-Cuartin G, Chalmers JD, Sibila O. Diagnostic challenges of bronchiectasis. Respir Med. 2016;116:70-77.

27. Yamamoto C, Yoneda T, Yoshikawa M, et al. Airway inflammation in COPD assessed by sputum levels of interleukin-8. Chest. 1997; 112(2):505-510.

28. Ji J, von Scheele I, Bergstrom J, et al. Compartment differences of inflammatory activity in chronic obstructive pulmonary disease. Respir Res. 2014;15:104-113.

29. Su B, Liu T, Fan H, et al. Inflammatory markers and the risk of chronic obstructive pulmonary disease: a systematic review and meta-analysis. PLoS One. 2016;11(4):e0150586.

30. Tsybikov NN, Egorova EV, Kuznik BI, Fefelova EV, Magen E. Anticytokine autoantibodies in chronic rhinosinusitis. Allergy Asthma Proc. 2015;36(6):473-480.

31. Hurst JR, Wilkinson TM, Perera WR, Donaldson GC, Wedzicha JA. Relationships among bacteria, upper airway, lower airway, and systemic inflammation in COPD. Chest. 2005;127(4):1219-1226.

32. Angrill J, Agusti C, De Celis R, et al. Bronchial inflammation and colonization in patients with clinically stable bronchiectasis. Am J Respir Crit Care Med. 2001;164(9):1628-1632.

33. Ayhan G, Tas D, Yilmaz I, et al. Relation between inflammatory cytokine levels in serum and bronchoalveolar lavage fluid and gene polymorphism in young adult patients with bronchiectasis. $J$ Thorac Dis. 2014;6(6):684-693.

34. Saha S, Brightling CE. Eosinophilic airway inflammation in COPD. Int J Chron Obstruct Pulmon Dis. 2006;1(1):39-47.

35. Siva R, Green RH, Brightling CE, et al. Eosinophilic airway inflammation and exacerbations of COPD: a randomized controlled trial. Eur Respir J. 2007;29(5):906-913.

36. Jin J, Liu X, Sun Y. The prevalence of increased serum IgE and Aspergillus sensitization in patients with COPD and their association with symptoms and lung function. Respir Res. 2014;15:130-142. 
37. Jin J, Yu W, Li S, Lu L, Liu X, Sun Y. Factors associated with bronchiectasis in patients with moderated-severe chronic obstructive pulmonary disease. Medicine. 2016;95(29):e4219.

38. Kroegel C, Schuler M, Forster M, Braun R, Grahmann PR. Evidence for eosinophil activation in bronchiectasis unrelated to cystic fibrosis and bronchopulmonary aspergillosis: discrepancy between blood eosinophil counts and serum eosinophil cationic levels. Thorax. 1998;53(6):498-500.

39. Koller DY, Urbanek R, Gotz M. Increased degranulation of eosinophil and neutrophil granulocytes in cystic fibrosis. Am J Respir Crit Care Med. 1995;152(2):629-633.
40. Hamilos DL. Drivers of chronic rhinosinusitis: inflammation versus infection. J Allergy Clin Immunol. 2015;136(6):1454-1459.

41. Guan WJ, Gao YH, Xu G, et al. Sputum matrix metalloproteinase-8 and -9 and tissue inhibitor of metalloproteinase-1 in bronchiectasis: clinical correlates and prognostic implications. Respirology. 2015;20(7):1073-1081.

\section{Publish your work in this journal}

The International Journal of COPD is an international, peer-reviewed journal of therapeutics and pharmacology focusing on concise rapid reporting of clinical studies and reviews in COPD. Special focus is given to the pathophysiological processes underlying the disease, intervention programs, patient focused education, and self management protocols.

\section{Dovepress}

This journal is indexed on PubMed Central, MedLine and CAS. The manuscript management system is completely online and includes a very quick and fair peer-review system, which is all easy to use. Visit $\mathrm{http}: / / \mathrm{www}$. dovepress.com/testimonials.php to read real quotes from published authors.

\footnotetext{
Submit your manuscript here: http://www.dovepress.com/international-journal-of-chronic-obstructive-pulmonary-disease-journal
} 